\title{
METHODOLOGY FOR DISPLAYING CCTV COVERAGE IN EXISTING VIDEO MANAGEMENT SYSTEM
}

\author{
Taewoo Kim ${ }^{1}$, Hyungheon $\mathrm{Kim}^{2}$, Pyeongkang $\mathrm{Kim}^{1}$ and Youngkyun $\mathrm{Cha}^{2}$ \\ ${ }^{1}$ Technology Laboratory, Innodep Inc., Seoul City, Korea \\ ${ }^{2}$ Graduate School of Information Security, Korea University, Seoul City, Korea
}

\begin{abstract}
CCTV is becoming more important in solving incidents. Various solutions have been developed for effective control, and GIS solution that displays CCTV video on the map is the most useful among them. On the other hand, in the current system, since CCTV does not have a sensor such as a digital compass, it displays only the location of CCTV, not the area that CCTVs view. In this paper, we present a methodology to indicate which area a CCTV is monitoring in the existing system. It can be accomplished by showing video regarding specific PTZ values to users and receiving information from users about the area.
\end{abstract}

\section{KEYWORDS}

PTZ Region, GIS, Parameter Estimation

\section{INTRODUCTION}

As the demand for safety increases, a large number of CCTVs are installed in various parts of the city, and these images play an important role in solving various crimes, incidents and accidents [1].

Usually, so many CCTVs are managed by a small number of monitoring agents, so various solutions have been developed to help them [2]. One of the most useful of these is the GIS solution. GIS solutions display the location where CCTV is installed and also provide the function to play the video of the selected CCTV. On the other hand, since most cameras don't have sensors like compasses, you can't know which direction in the map you are looking by just playing video, or it takes much time and effort to recognize the direction of the camera.

In emergency situations, it is very important to know exactly where the video is happening on the map. By the way, if the system knows which area each camera is currently viewing, it will be possible to control the camera for viewing the exact region to cope with the situation. It will also be possible to search for videos related to a specific area, resulting in efficiency of monitoring [3]. On the other hand, almost all existing cameras don't yet have a bearing-like sensor, like a compass, so it's not easy to know which direction the camera is looking at. It can be solved by Natarajan Meghanathan et al. (Eds) : ACITY, AIAA, DPPR, CNDC, WIMNET, WEST, ICSS - 2019 pp. 97-102, 2019. (C) CS \& IT-CSCP 2019

DOI: $10.5121 /$ csit.2019.91710 
attaching a compass to an existing camera, but since each camera has various physical structures, it is difficult to install a compass for each camera.

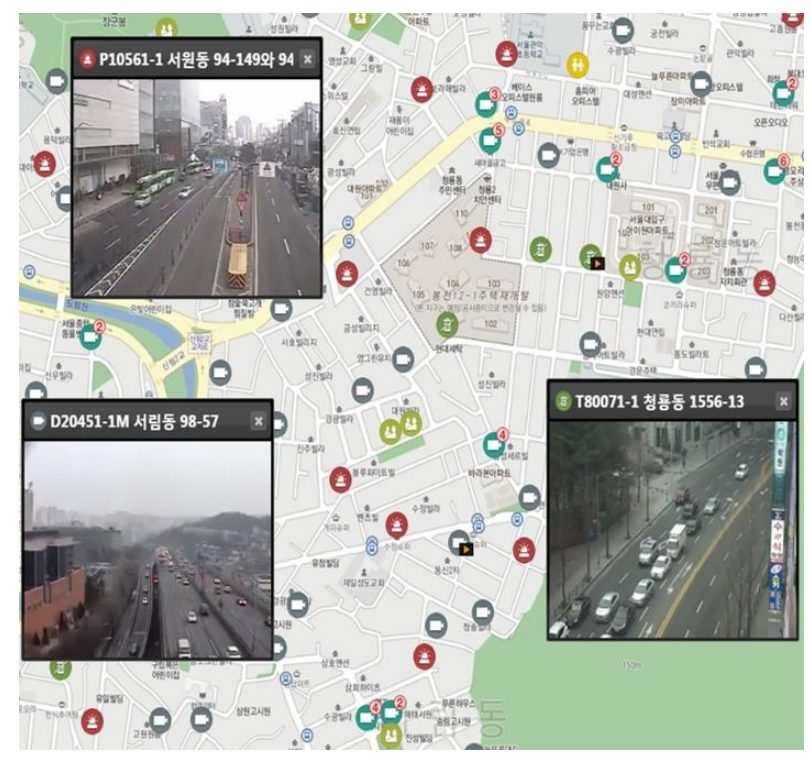

Figure 1. GIS solution example playing CCTV video

In order to solve this problem, this paper proposes a method to display the camera viewed region on the map using the existing system, which is similar in [4]. Chapter 2 describes the methodology for the system to calculate the bearing. Chapter 3 shows the results of the implementation and concludes this paper in Chapter 4.

\section{Methodology}

The proposed method displays not only the position of the camera but also the area monitored by the camera as shown in the Figure 2. It is divided into two steps: estimating unknown parameters and calculating ptz values by which the camera is controlled to view selected region. The key to this methodology is to show the user an arbitrary cctv video whose ptz value is known and to get user to indicate where the region is. Then with this information the unknown parameters like the height of the installed cctv, the vertical field of view(FOV) and the horizontal field of view is acquired. 
Computer Science \& Information Technology (CS \& IT)

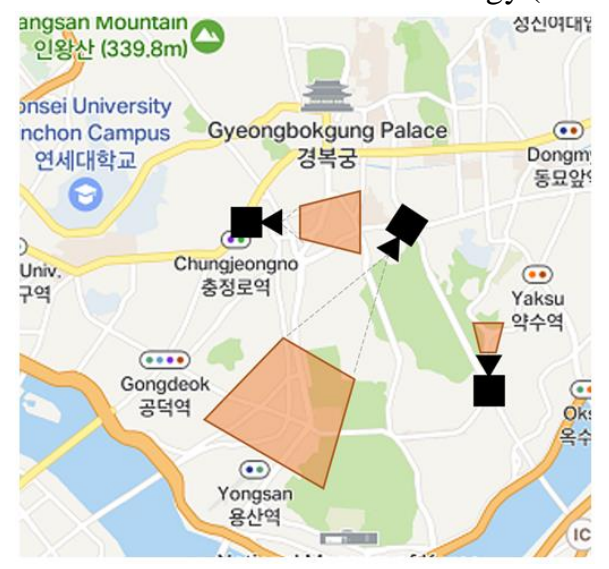

Figure 2. The map concept indicating the monitored region

\subsection{Estimation of Unknown Parameters}

Unknown parameters are the installation height of the camera, the vertical FOV angle of the camera and the horizontal FOV angle of the camera. The vertical FOV of the camera represents the vertical angle of view of the camera lens, and the horizontal FOV represents the horizontal angle of view. Overall parameters are illustrated in figure 3. In figure 3, the monitored area is represented as a trapezoid, which means that the farther area shows the wider area on the map because the camera has a constant FOV angle. and the horizontal FOV represents the horizontal angle of view. In addition, the other unknown variables are the ptz values. In order to find all these unknowns, the method controls the camera with a certain pan, tilt value with zoom level 1 in the system and then shows the image to the user.
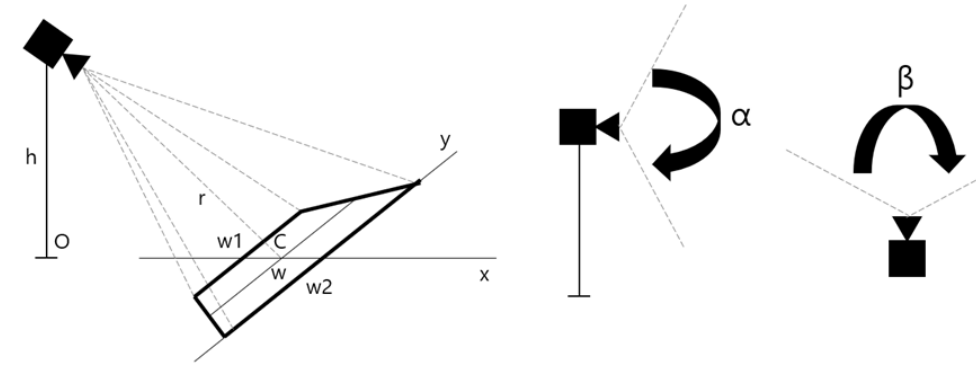

Figure 3. Parameters related to viewing a region

The user indicates which area on the map corresponds to the area visible in the video. When the user indicates the area, it means that the distance of the monitored area from the camera and the horizontal length of the monitored area are known. This is shown in the figure 4 . The length $\mathrm{d} 1$ corresponds to the distance from the camera to the nearest position shown in the image, and w1 corresponds to the horizontal distance of the nearest horizontal line. In fact, the given values are the coordinates of the four vertices of the trapezoid, which can be used to know d1, d2, w1, and w2. From the know values, the unknows $h, \alpha$ and $\beta$ can be found by using following equations. In equation, $\varphi$ means tilt angle of the camera. 


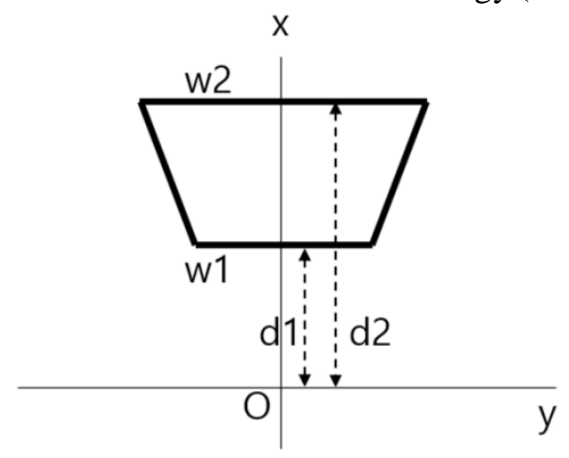

Figure 4. Dimensions of selected trapezoid region

$$
\begin{aligned}
& \beta=2 * \arctan (\mathrm{w} 2 / \mathrm{d} 2 / 2) \\
& \mathrm{d} 1=\mathrm{h} * \tan (\varphi-\alpha / 2) \\
& \mathrm{d} 2=\mathrm{h} * \tan (\varphi+\alpha / 2)
\end{aligned}
$$

The values $h$ and $\alpha$ are acquired by solving the system of equations. This process is repeated three to four times and is determined by averaging the $h, \alpha$ and $\beta$ values obtained in each case.

\subsection{Calculation of Ptz for Region Selected by user}

Since $h, \alpha$ and $\beta$ values are obtained in the forgoing process, when the user wants to control the area using the GUI tool, the system can determine how to adjust the ptz values to steer the area by the equations from (5) to (7). The parameters related to calculation are drawn in the figure 5. The d1 represents the distance from the camera to the nearest point of selected region and $w 3$ is the shorter width of the trapezoid. The $\theta$ is the delta pan angle to be added to the initial pan angle. The objective parameters are $\mathrm{p} 2, \mathrm{t} 2$ and zoom.

$$
\begin{gathered}
\theta=\arccos ((\mathrm{x} 1 * \mathrm{y} 1+\mathrm{x} 2 * \mathrm{y} 2) / \operatorname{norm}((\mathrm{x} 1, \mathrm{y} 1)) / \operatorname{norm}((\mathrm{x} 2, \mathrm{y} 2)) \\
\mathrm{p} 2=\mathrm{p} 1+\theta \\
\mathrm{t} 2=\arctan (\mathrm{d} 3 / \mathrm{h})+\alpha / 2 \\
\mathrm{zoom}=2 * \mathrm{~d} 3 * \tan (\beta / 2) / \mathrm{w} 3
\end{gathered}
$$




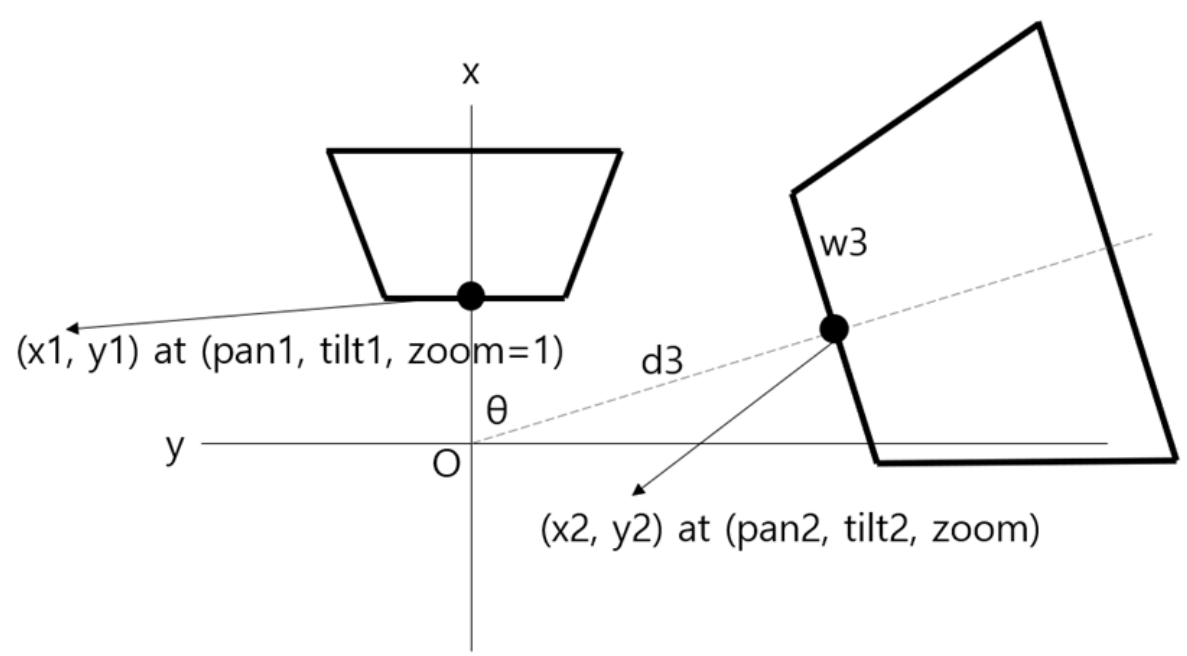

Figure 5. Calculation of ptz for selected region

\section{Simulation ReSUlt}

In order to implement the proposed methodology, the camera is installed in the office. In general, the ptz camera is installed upside down on the pole and is developed to look downward. Since the equipment for mounting the camera is very expensive, the simulation assumes that the office ceiling is the ground. Figure 6 shows the appearance of controlling ptz.
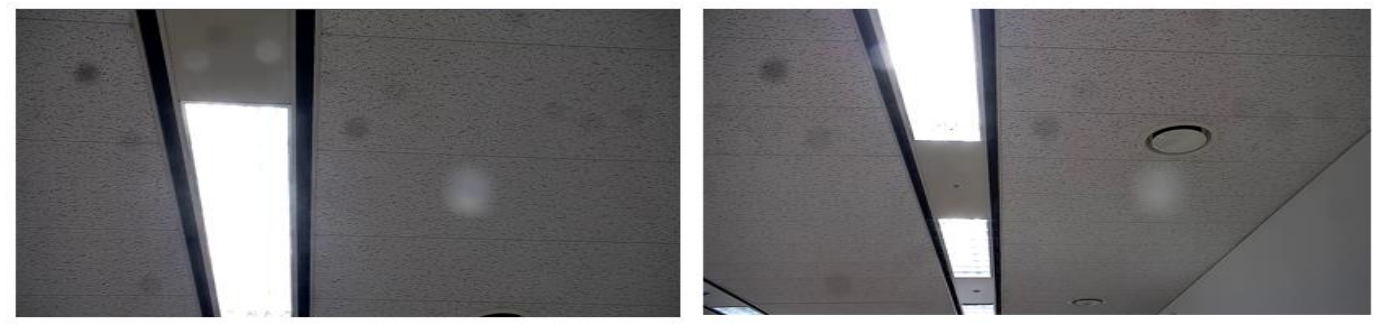

Figure 6. PTZ control result

\section{Conclusions}

As the importance of the integrated control center increases, how to effectively use the control information becomes important In addition to displaying a simple location of cctv on the map, recording information on which areas are being controlled can also improve control efficiency. This becomes possible in existing system using proposed method. In this paper, we presented a methodology that can display the control area without introducing additional equipment to the existing system through user's supervised learning, and the methodology using a real camera. It is hoped that the proposal of this paper can improve the control efficiency. 


\section{ACKNOWLEDGEMENTS}

This work was supported by Institute for Information \& communications Technology Promotion(IITP) grant funded by the Korea government(MSIT) (2017-0-00250, Intelligent Defense Boundary Surveillance Technology Using Collaborative Reinforced Learning of Embedded Edge Camera and Image Analysis)

\section{REFERENCES}

[1] V. S. Asirvadam, C. P. Fen, and N. Saad, P. Sebastian, A. A. Altahir, A. Ali, N. H. Hamid, "Risk map for video surveillance CCTV," 2014 ICIAS, June 2014.

[2] G. Thiel, "Automatic CCTV surveillance-towards the VIRTUAL GUARD," Proceedings IEEE 33rd Annual 1999 International Carnahan Conference on Security Technology, Oct. 1999.

[3] Walters, "CCTV operator performance and system design," 1993 Proceedings of IEEE International Carnahan Conference on Security Technology, Oct 1993.

[4] A. A. Altahir, V. S. Asirvadam, N. B. Hamid, P. Sebastian, "Modeling camera coverage using imagery techniques for surveillance applications," IEEE International Conference on Control System, Computing and Engineering, Nov 2014. 